\title{
BIVALENCIA, FATALISMO E INACCIÓN EN CRISIPO
}

Ricardo SALLES

Instituto de Investigaciones Filosóficas Universidad Nacional Autónoma de México rsalles@servidor.unam.mx

RESUMEN: Este ensayo ofrece un análisis del argumento de Crisipo a favor de que todo tiene una causa en Cicerón, De Fato 20. Para ello, se discute en qué sentido el argumento es fatalista y si el tipo de fatalismo que implica alienta la inacción. Asimismo, se presenta una nueva interpretación de la réplica de Crisipo al Argumento Perezoso en Eusebio, Praep. ev. 6.8.28. En particular se sostiene que, para Crisipo, la relación entre sucesos codestinados es analítica: a fin de determinar qué sucesos futuros están codestinados con sucesos presentes, basta analizar los conceptos que se emplean para describir los primeros.

PALABRAS CLAVE: estoicismo, motivación para actuar, determinismo

SUMMARY: In this paper I undertake an examination of Chrysippus' argument in Cicero's De Fato 20 for the view that everything has a cause, by discussing in what sense it is fatalist and whether the kind of fatalism it implies encourages idleness. A novel interpretation is offered of Chrysippus' refutation of the Idle Argument at Eusebius, Praep. ev. 6.8.28. In particular, I argue that for Chrysippus the connection between co-fated events is analytic: to determine which future events are co-fated with present ones, it is sufficient to analyse the concepts that are used to describe the former.

KEY WORDS: stoicism, motivation, determinism

El propósito de este trabajo es reconstruir uno de los elementos del argumento que ofreció Crisipo - tercer escolarca de la escuela estoica - a favor del determinismo, entendido como la tesis de que es causalmente necesario todo estado y suceso que se da. ${ }^{1}$

Crisipo infiere esta conclusión a partir de dos premisas: (a) todo estado y suceso tiene una causa, y (b) en toda relación

${ }^{1}$ Crisipo no fue el primer estoico en defender esta última tesis. En palabras de Zenón de Citio (334-262 a.C.), el fundador de la escuela: "es imposible que una causa se haga presente, pero que no se dé aquello de lo cual es causa". Cfr. Zenón ap. Estobeo, E 1.138,14-139,4 (SVF 1.89, 2.336; LS 55A):

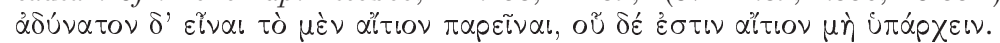


causal, la causa es condición suficiente para que se dé el efecto, el cual, por lo tanto, es necesario si se da su causa. ${ }^{2}$ La inferencia es deductivamente válida. Si se da un estado o suceso $E$, (a) implica que $E$ tiene una causa $C$; y dado que $C$ causa $E$, se sigue de (b) que $E$ es causalmente necesario dado $C$, pues $C$ es suficiente para que se dé $E$. Por lo tanto, para establecer la necesidad causal de un estado o suceso a partir de (a) y (b), sólo se necesita la tesis empírica de que dicho estado o suceso efectivamente se da. Ninguna de estas dos tesis, sin embargo, es suficiente por sí sola para establecer la conclusión y cada una es lógicamente independiente de la otra. En efecto, (a) sin (b) es compatible con la afirmación de que ningún estado o suceso es causalmente necesario, esto es, que ninguno se sigue de otro por necesidad causal, mientras que (b) sin (a) es compatible con la idea de que no todo estado o suceso es causalmente necesario. Para dar con la conclusión determinista a la que Crisipo pretende llegar, se necesitan ambas tesis. Asimismo, dada su mutua independencia lógica, la solidez del argumento requiere dos argumentos específicos: uno a favor de (a) y otro a favor de (b).

En este trabajo me ocuparé del argumento específico que adujo Crisipo en defensa de la tesis (a). Veremos que se basa en la aplicación del principio de bivalencia a proposiciones que se refieren al futuro, lo cual desemboca en una cierta forma de fatalismo y, por consiguiente, plantea el problema de la razonabilidad de esforzarse por alcanzar los objetivos que nos hemos propuesto: si el futuro está fijado de antemano y los sucesos

${ }^{2}$ Aquí cabe hacer dos aclaraciones. En primer lugar, en este contexto lo que se entiende por "causa" no es ninguno de los elementos del conjunto de factores cuya conjunción produjo el efecto (los cuales, como se indica en Mackie 1965, pp. 15-16, suelen ser individualmente insuficientes para producir por sí solos el efecto), sino el conjunto mismo de esos factores. Por otra parte, no debe confundirse la tesis (b) ni con el fatalismo que llamo "incoherente" ( $c f r$. Sec. 2), según el cual todo suceso habría ocurrido necesariamente aun cuando no se hubiera dado aquello que efectivamente lo causó (lo cual supone que la causa, a diferencia, del efecto, es contingente) ni con el fatalismo que llamo "no causal" ( $c f r$. Sec. 2), según el cual no existe un vínculo realmente explicativo entre una causa y su efecto. Como veremos, Crisipo combatió estas dos formas de fatalismo. 
que habrán de ocurrir son, por ello, inevitables, ¿qué sentido tiene esforzarse por alcanzar cualquier cosa? La respuesta de Crisipo será que el fatalismo estoico, a diferencia de otros tipos de fatalismo, no conduce a la inacción.

\section{Bivalencia, verdad futura y causalidad}

Antes de investigar por qué Crisipo pensaba que todo tenía una causa, vale la pena examinar brevemente algunos elementos de la teoría estoica de la causalidad.

A grandes rasgos, la causalidad estoica funciona de la forma siguiente: $^{3}$ (i) las relaciones causales se dan entre objetos corpóreos y, en toda relación causal, uno de los cuerpos es causa de que otro satisfaga un predicado (e.g. el cuchillo es causa de que la carne satisfaga el predicado "ser cortada"); (ii) cuando un cuerpo $A$ actúa causalmente sobre un cuerpo $B$, los estoicos dicen que el efecto propio de la actividad de $A$ es el predicado satisfecho por $B{ }^{4}$ aunque esto puede significar simplemente que el efecto no es tanto el predicado mismo, sino el que $B$ satisfaga el predicado; por último, (iii) esta satisfacción suele ser un suceso: el deseo de Dión de caminar causó que el predicado "caminar" sea satisfecho por él, donde su caminar es un suceso; aunque también puede ser un estado: la acción del cuchillo sobre mi carne causó que el predicado "estar dolorido" fuese satisfecho por mí, donde estar dolorido es un estado. ${ }^{5}$ Los estoicos también sostenían que toda causa es activa. A fin de que $A$ cause que un predicado $P$ sea satisfecho por $B, A$ mismo tiene que pasar por alguna actividad o cambio, y $A$ tiene esta eficacia causal en virtud de tal actividad o cambio. Por lo tanto, cualquier cuerpo que actúe como una causa debe él mismo satisfacer un predicado que exprese una actividad. A fin de que el cuchillo cause que la carne satisfaga el predicado "ser cortada", debe participar en una actividad de corte y así satisfacer el predicado "cortar". Con ello, los estoicos negaron el estatus de causa a los motores inmóviles, los cuales, según Aristóteles,

${ }^{3}$ Cfr. Frede 1987, Duhot 1989 y Bobzien 1999.

${ }^{4}$ Cfr. E 1.138, 14-15 (SVF 1.89, 2.336; LS 55A).

${ }^{5}$ Cfr. $M 9.211$ (SVF 2.341; LS 55B). 
eran el paradigma de un cierto tipo de causa, a saber, la final. Por el momento, sólo necesitamos tener presente que en boca de Crisipo "todo estado o suceso tiene una causa" significa que, cuando un predicado es satisfecho por un cuerpo, tiene que haber una causa de que sea satisfecho por ese cuerpo.

Como señalé en la introducción, para mostrar que efectivamente todo estado o suceso tiene una causa, Crisipo empleó un argumento basado en el principio de bivalencia, a saber, el principio:

(PB) Para cualquier proposición $P, P$ es o verdadera o falsa.

El argumento fue recogido por Cicerón en el párrafo 20 de su tratado De Fato:

Si hay movimiento sin causa, entonces no ocurre que toda proposición (lo que los dialécticos llaman un axioma) sea o verdadera o falsa. Pues lo que no tenga alguna causa eficiente que lo provoque no será ni verdadero ni falso. Pero toda proposición es verdadera o falsa; de modo que no hay movimiento sin causa. ${ }^{6}$

Como puede apreciarse, el argumento se centra en sucesos, o "movimientos", pero, mutatis mutandis, también puede aplicarse a estados. En esencia, procede de la siguiente forma: (i) si

6 "Si est motus sine causa, non omnis enuntiato (quod $\alpha \xi i \omega \mu \alpha$ dialectici appellant) aut vera aut falsa erit. causas enim efficientis quod non habebit, id nec verum nec falsum erit. omnis autem enuntiatio aut vera aut falsa est; motus ergo sine causa nullus est." Cfr. F 26: "Como esto es así, ¿por qué toda proposición no habría de ser o verdadera o falsa si no concedemos que cualquier cosa que sucede se debe al destino? 'Porque', él <sc. Crisipo> dice, 'aquellas cosas futuras que no tengan causas para su futuro ser no pueden ser verdaderas; de modo que aquellas cosas que son verdaderas necesariamente tienen causas; y por tanto cuando hayan sucedido, lo habrán hecho por obra del destino." ("Quod cum ita sit quid est, cur non omnis pronuntiatio aut vera aut falsa sit nisi concesserimus fato fieri, quaecumque fiant? Quia futura vera, inquit, non possunt esse ea quae causas cur futura sint non habent; habeant igitur causas necesse eat ea, quae vera sunt; ita, cum evenerint, fato evenerint.") Sobre la aplicación de (PB) a las proposiciones en general, véanse DL 7.65 (SVF 2.193; LS 34A); Cicerón, acad. 95 (SVF 2.196; LS 37H5); $M$ 8.10 (SVF 2.195). Sobre su aplicación a proposiciones que se refieren al futuro en particular, véase Simplicio, in Cat. 406, 34-407, 3 (SVF 2.197). Sobre la otra premisa del argumento, véase nuevamente $F 26$. 
hubiera sucesos sin causa, $(\mathrm{PB})$ no valdría para proposiciones que se refieren al futuro; pero (ii) toda proposición (incluyendo aquellas que se refieren al futuro) es o verdadera o falsa; por consiguiente, (iii) no puede haber sucesos sin causa; de este modo, (iv) todo suceso tiene una causa. El argumento en su conjunto es válido en virtud de su forma lógica, que es la de un modus tollens. ${ }^{7}$ Su solidez final dependerá de que cada una de sus dos premisas sea verdadera o, al menos, mínimamente verosímil. En el pasaje de Cicerón no se dan razones a favor de ninguna de ellas. Para examinar su verosimilitud, es preciso investigar su significado.

Una consecuencia lógica de la segunda premisa es que cualquier proposición en futuro es ahora o verdadera o falsa, como de algún modo lo sugiere el texto: "toda proposición es o verdadera o falsa" (suponiendo que el "es" está dotado de temporalidad y se refiere al presente). Según esta interpretación, Crisipo no está sosteniendo la tesis trivial de que toda proposición que se refiere al futuro será o verdadera o falsa en el tiempo futuro indicado por su indicador temporal; para poner un ejemplo, que una proposición como Voy al cine el viernes a las cinco será o verdadera o falsa el viernes a las cinco. ${ }^{8}$ La idea es, más bien, que esta proposición, a pesar de no expresar un suceso presente, sino futuro, ya posee, en el presente, un valor de verdad. De modo general:

\footnotetext{
${ }^{7}$ Más precisamente es la de un modus tollendo tollens (si $A$, entonces $B$; pero no $B$; por lo tanto, no $A$ ), que se distingue del modus ponendo tollens (o bien $A$ o bien $B$ [la disyunción es excluyente]; pero $A$, por lo tanto, no $B)$. Sobre el desarrollo de estas reglas de inferencia en la lógica proposicional antigua, $c f r$. Bobzien 2002.

${ }^{8}$ En el ejemplo, "voy" se usa no temporalizadamente de modo que la proposición sea equivalente a Iré al cine el viernes a las cinco. Las proposiciones en futuro cuyos indicadores temporales están especificados por fechas definidas son equivalentes a las proposiciones no temporalizadas que incluyen a los indicadores (Iré al cine el viernes a las cinco es equivalente a Voy [no temporalizado] al cine el viernes a las cinco). Esto también vale para las proposiciones en futuro que incluyen indicadores temporales deícticos (Iré al cine mañana a las cinco es equivalente a Voy [no temporalizado] al cine mañana a las cinco). Para una discusión amplia de estas distinciones y su aplicabilidad a la filosofía antigua, $c f r$. Gaskin 1995.
} 
$\left(\mathrm{P}_{2}\right)$ Para cualquier proposición $P$ tal como $S$ ocurre en $t$, donde $S$ es un suceso y $t$ un tiempo futuro, $P$ ya es, en el presente, o verdadera o falsa.

Si nos atenemos a la letra del texto de Cicerón, Crisipo no pretende inferir la necesidad de una proposición que se refiere al futuro, a partir de su verdad presente (ni su imposibilidad a partir de su falsedad). En otras palabras, no hay nada en el texto que pudiera sugerir las tesis $\left(\mathrm{P}_{n}\right)$ y $\left(\mathrm{P}_{i}\right)$ :

$\left(\mathrm{P}_{n}\right)$ Para toda proposición $P$ de la forma $S$ ocurre en $t$, donde $t$ es un momento futuro, si $P$ ya es verdadera, $P$ es necesaria ahora o en algún otro momento; esto es, es necesario ahora (o en algún otro momento) que $S$ ocurra en $t$.

$\left(\mathrm{P}_{i}\right)$ Para toda proposición $P$ de la forma $S$ ocurre en $t$, donde $t$ es un momento futuro, si $P$ ya es falsa, $P$ es imposible ahora (o en algún otro momento); esto es, es imposible ahora (o en algún otro momento) que $S$ ocurra en $t$.

En general, el blanco de ataque de las objeciones filosóficas en contra de la aplicación de (PB) a las proposiciones que se refieren al futuro no es tanto la idea misma de que dichas proposiciones pudieran ya ser verdaderas o falsas, como el posible vínculo que su verdad o falsedad anticipada pueda tener con la predeterminación, como lo sugieren $\left(\mathrm{P}_{n}\right)$ y $\left(\mathrm{P}_{i}\right)$. Aristóteles, por ejemplo, según una lectura que se ha hecho de int. 9, rechaza la aplicación de (PB) a estas proposiciones en el supuesto de que $s i$ se aplicara a ellas, también tendría que concederse la predeterminación. ${ }^{9}$ Pero de hecho la verdad (o la falsedad) anticipada y la predeterminación son dos afirmaciones independientes, la primera de las cuales no tiene por qué implicar la segunda para ser

\footnotetext{
${ }^{9}$ En la época moderna se pueden encontrar versiones de esta interpretación en Ross 1924, vol. 1, p. lxxxi, Quine 1953, Lukasiewicz 1967, Wieland 1979 y Sorabji 1980, cap. 5. Hay una interpretación rival: Aristóteles concede la verdad o la falsedad anticipada, pero niega la inferencia de su oponente de la verdad o la falsedad anticipada a la predeterminación. Cfr. Anscombe 1956, Williams 1980 y Lowe 1980.
} 
coherente. ${ }^{10}$ En tal caso, Crisipo no está adoptando una posición incoherente en $F 20$ si, al mismo tiempo que sostiene $\left(\mathrm{P}_{2}\right)$, no suscribe las tesis $\left(\mathrm{P}_{n}\right)$ y $\left(\mathrm{P}_{i}\right)$, las cuales afirman que la verdad o la falsedad anticipada conlleva por sí misma predeterminación.

La originalidad del argumento de Crisipo reside en su primera premisa: "si hubiera sucesos sin causa, el principio de bivalencia no se aplicaría a las proposiciones en futuro". Las proposiciones que carecerían de valor de verdad si hubiese sucesos sin causa serían aquellas que describen los sucesos en cuestión y que afirman su ocurrencia en algún tiempo futuro. ${ }^{11}$ Una formulación precisa de la primera premisa sería entonces:

$\left(\mathrm{P}_{1}\right)$ Para cualquier suceso $S$ que ocurra sin causa en algún tiempo futuro $t$, la proposición $S$ ocurre en $t$ no es aún ni verdadera ni falsa.

Aun cuando en Cicerón no se ofrezca ningún argumento a favor de $\left(\mathrm{P}_{1}\right)$, podemos conjeturar que detrás de la tesis estaban las siguientes razones. ${ }^{12}$ En el ejemplo usado en $\left(\mathrm{P}_{1}\right)$, la afirmación de que la proposición no es falsa se basa probablemente en la idea de que para toda proposición que afirme la ocurrencia de un suceso, su falsedad exige la no ocurrencia del suceso. ${ }^{13}$ Así, dado que, según $\left(\mathrm{P}_{1}\right)$, el suceso sí ocurre, la proposición no puede ser falsa. Ahora bien, ¿por qué tampoco puede ser verdadera? Una razón posible sería la siguiente:

$\left(\mathrm{T}_{c}\right)$ Para cualquier suceso $S$ que ocurre en algún tiempo futuro $t$, la proposición $S$ ocurre en $t$ no puede ser verdadera ahora, a menos que haya una causa ahora de que $S$ ocurra en $t$ (y, por ende, una cadena causal que abarque desde el presente hasta el momento futuro $t$ ).

${ }^{10}$ Cfr. Hintikka 1964, esp. las pp. 487-488. Véanse también Bosley 1977, Fine 1984 y Gaskin 1995, p. 15. McCall explica detalladamente la propuesta de Hintikka (manteniendo una posición contraria) en la sección 5 de McCall 1966.

${ }^{11}$ Véase Bobzien 1999, pp. 63-65.

${ }^{12}$ Sigo en algunos aspectos la reconstrucción desarrollada en McCall 1966, pp. 276-277 y Bobzien 1998, pp. 65-71.

${ }^{13}$ Los estoicos compartieron esta tesis. Véase $M$ 8.10. 
Se atribuye a Crisipo una versión de $\left(\mathrm{T}_{c}\right)$ en $F$ (26): "aquellas cosas futuras que < ahora $>$ no tengan causas para su futuro ser no pueden ser verdaderas; de modo que aquellas cosas que son verdaderas necesariamente tienen causas <ahora>". ${ }^{14}$ Y una razón para $\left(\mathrm{T}_{c}\right)$ podría ser la siguiente. La proposición en tiempo futuro $S$ ocurre en $t$ no puede ser verdadera ahora si no hay nada en el presente que garantice su verdad, pero nada en el presente podría garantizar su verdad más que el hecho de que algún suceso presente $S p$ es causa de $S$ (eventualmente a través de la mediación de una serie de sucesos $\left\langle\mathrm{S}^{\prime}, \mathrm{S}^{\prime \prime}, \ldots\right\rangle$ tal que la serie $<S p, \mathrm{~S}^{\prime}, \mathrm{S}^{\prime \prime}, \ldots, \mathrm{S}>$ forma una serie ordenada en la cual cada miembro es causa del siguiente). Dicho de otro modo, la verdad presente de $S$ ocurre en $t$ parece requerir que el suceso $S$ esté "presente en sus causas". Por ejemplo, la proposición Voy al cine el viernes a las cinco no puede ser verdadera ahora a menos que las causas del suceso ya estén presentes de algún modo, por ejemplo, si tengo el deseo ahora de ir al cine el viernes a las cinco y no hay obstáculos externos para mi deseo que pudieran impedirlo. Así pues, si suponemos que $S$ carece de una causa, entonces, dado que no puede satisfacerse la condición necesaria enunciada en $\left(\mathrm{T}_{c}\right)$ para la verdad de la proposición, ésta no puede ser verdadera ahora. Mutatis mutandis, el mismo argumento se aplica a la falsedad de una proposición.

Para recapitular, es sobre la base de $\left(\mathrm{T}_{c}\right)$ que Crisipo infiere la causalidad a partir de la verdad anticipada. Esta inferencia, a su vez, explica por qué es verdadera $\left(\mathrm{P}_{1}\right)$ - la primera premisa del argumento de Crisipo: "si hubiera sucesos sin causa, las proposiciones en futuro carecerían de valor de verdad"- y, en particular, por qué no pueden ser verdaderas (o falsas) las proposiciones que se refieren al futuro y que expresan sucesos sin causa. Por su parte, $\left(\mathrm{P}_{1}\right)$, al combinarse con la segunda premisa del argumento - "las proposiciones en futuro ya son o verdaderas o falsas"-, implica que ningún suceso futuro carece de causa. Podemos generalizar la tesis a todos los tiempos, que es el resultado que Crisipo pretendía alcanzar ("motus ergo

${ }^{14}$ Quia futura vera non possunt esse ea quae causas cur futura sint non habent; habeant igitur causas necesse eat ea, quae vera sunt. 
sine causa nullus est"). En efecto, dado que en el pasado lo que ahora es presente estaba en el futuro y lo que es ahora pasado fue presente, entonces, para todo suceso actualmente presente había en el pasado una proposición verdadera que, en ese entonces, aseveraba su ocurrencia; en consecuencia, había en ese tiempo una causa de su (entonces) futura ocurrencia; de este modo, todo suceso del presente tiene una causa; por paridad de razonamiento, todo suceso del pasado tiene una causa; en consecuencia, todo suceso (pasado, presente y futuro) tiene una causa.

\section{El fatalismo y el problema de la inacción}

La idea de que el futuro está predeterminado fue objeto de críticas severas por parte de filósofos antifatalistas, empezando por Aristóteles. A él le debemos la objeción de que el fatalismo conduce a la inacción, pues implicaría que no es razonable esforzarse por alcanzar ningún objetivo. Crisipo intentó salvar al fatalismo de esta objeción, mostrando que no conduce necesariamente a esta consecuencia. En esta sección me propongo señalar cuál es el argumento que él desarrolló para establecer esto. Como veremos, éste consiste en trazar una distinción sustantiva entre, por una parte, el fatalismo no causal, que Aristóteles ataca y que conduce efectivamente a la inacción, y, por otra, el fatalismo causal, el cual Crisipo identifica con la única versión correcta del fatalismo y que, entre otras cosas, no conduce a la inacción. Esta distinción representa una de las contribuciones más significativas de la filosofía griega a la teoría determinista. Veamos primero la objeción antifatalista de Aristóteles.

La objeción de Aristóteles figura en int. 9 y se centra en la deliberación. Por "deliberación" se entiende un razonamiento práctico que parte de una meta deseada y emprende la búsqueda retroactiva de los medios para alcanzarla. Como resultado de una deliberación, elegimos y emprendemos las acciones que, según nuestro razonamiento, conducen a la meta. ${ }^{15} \mathrm{La}$ objeción antifatalista aparece en 18b30-31: si "todo <en el futuro> es y

${ }^{15}$ Cfr. en particular EN 3.21111 b26-29. Hay otros pasajes igualmente significativos. Véanse, por ejemplo, 3.2 1112a15-17, $3.31113 \mathrm{a} 2-9$ y 6.3 1139a31- 


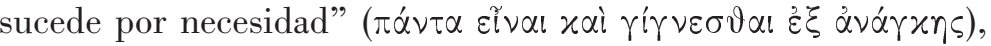
entonces,

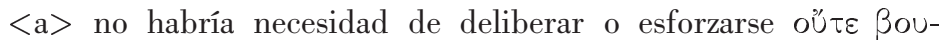

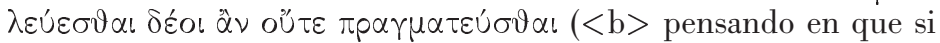
hacemos esto, aquello sucederá, pero si no lo hacemos, entonces no sucederá).

El texto merece mucha atención. La observación entre paréntesis en $\langle\mathrm{b}\rangle$ asevera que deliberamos basándonos en la suposición de que "si hacemos esto, aquello sucederá, pero si no lo hacemos, entonces no sucederá". La suposición no es sólo que nuestra deliberación provoque ciertas acciones ("si hacemos esto $<$ i.e. deliberar>, esto sucederá": $\mathrm{p} \rightarrow \mathrm{q}$ ), sino también que la primera es una condición sine qua non para la segunda ("pero si no lo hacemos, entonces no sucederá": $\neg p \rightarrow \neg q$, lo que da por contraposición $q \rightarrow p$ ). Dicho de otra manera, deliberamos y nos esforzamos teniendo como suposición que nuestra deliberación y la molestia que nos tomamos son suficientes y necesarias para sus consecuencias reales. ${ }^{16}$ Ahora bien, de acuerdo con $\left.<\mathrm{a}\right\rangle$, "no habría necesidad de deliberar" si el futuro estuviera predeterminado. Si estoy enfermo y, como resultado de haber deliberado, consulto a un médico acerca de cómo curarme, mi deliberación puede haber sido una condición suficiente para mi acción, pero no una condición necesaria: como estaba predeterminado a consultar a un médico, habría consultado a un médico aun cuando no hubiese deliberado. Por lo tanto, la predeterminación no sólo hace que nuestras deliberaciones sean superfluas: también quebranta nuestra suposición de que no lo son. Encontramos una versión de esta objeción en el peripatético Alejandro de Afrodisia, unos quinientos años después:

bl3. Al respecto, cfr. Sorabji 1973-1974, pp. 203-204 y 206, y Wiggins 1975, pp. 227-231.

${ }^{16}$ La tesis de la suficiencia debe entenderse en el contexto de la teoría aristotélica de la deliberación en la EN (cfr. especialmente 3.3 1112a30-31), según la cual sólo es razonable deliberar sobre cómo alcanzar algo que depende de nosotros ( $\hat{\varepsilon} \varphi^{\prime} \dot{\eta} \mu \tilde{\imath} \nu<\grave{\varepsilon} \sigma \tau \iota>$ ); esto, según Aristóteles, excluye que algo me impida alcanzar la meta $\varphi$ si mi deliberación es razonable y me indica que $\psi$ sí conduce a $\varphi$. 
Pero si hiciéramos todo lo que hacemos por ciertas causas establecidas de antemano, de modo que no tenemos la facultad de hacer esto en particular o no (...), ¿qué ventaja nos aporta, en lo que concierne a la acción, deliberar acerca de lo que hemos de hacer? Pues < según este punto de vista> es necesario que, aun después de haber deliberado, hagamos lo que habríamos hecho si no hubiéramos deliberado, de modo que no sacamos ninguna ventaja de deliberar más allá del hecho mismo de haber deliberado. ${ }^{17}$

La objeción aristotélica, sin embargo, ya era un tema de debate filosófico en el periodo helenístico, cuando se conocía

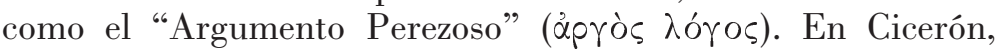
F 28-29, se expone una versión importante de él:

"Si está escrito que te recuperes de esta enfermedad, entonces te recuperarás, llames o no llames a un médico; igualmente, si está escrito que no te recuperes de esta enfermedad, entonces no te recuperarás, llames o no llames al médico. Pero lo uno o lo otro está predestinado, de modo que no tiene caso llamar al doctor." Este tipo de argumento se llama correctamente perezoso y ocioso, pues por el mismo argumento toda actividad se retirará de la vida. Pues se puede cambiar el argumento de la siguiente manera para que no introduzca el nombre de "destino", y siga sosteniendo la misma posición: "Si esto ha sido verdad por toda la eternidad, que 'Te recuperarás de esta enfermedad', entonces te recuperarás, llames o no llames al doctor; y de igual modo, si esto ha sido falso por toda la eternidad, 'No te recuperarás de esta enfermedad', entonces no te recuperarás, llames o no llames al doctor"; y lo demás se sigue.

Antes de pasar a la respuesta de Crisipo, es preciso poner de manifiesto una ambigüedad de la propia objeción.

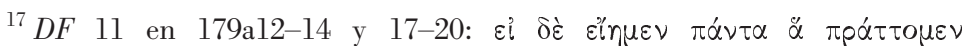

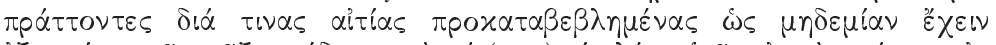

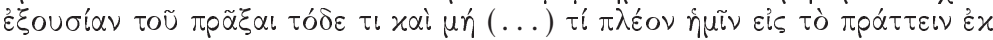

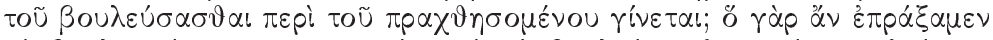

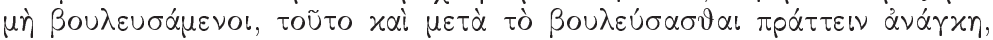

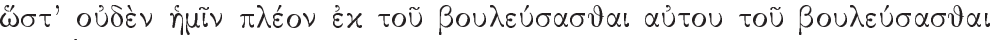

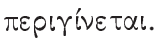


En sus tres versiones, la objeción admite dos lecturas considerablemente distintas, a las cuales me referiré como (1) y (2). Para un suceso presente $P$ y un suceso futuro predeterminado $F$ tales que $P$ y $F$ se relacionan aparentemente de modo causal,

(1) se sigue del fatalismo que $F$ habrá de darse aun cuando $P$ no se diera, en el entendido de que (a) $P$ es contingente (i.e. $P$ puede no darse en el presente), y de que (b) si $P$ no se diera, $F$ se daría de todas formas, lo cual es absurdo;

(2) se sigue del fatalismo que, si bien $P$ y $F$ son necesarios (ambos tienen que darse), $F$ no se dará porque se da $P$, esto es, no existe realmente una relación causal explicativa entre $F$ y $P$, lo cual es absurdo.

En ambas lecturas, la objeción es que, si el fatalismo fuera correcto, ningún suceso futuro predeterminado dependería de que se dieran los sucesos presentes que están aparentemente relacionados con él de modo causal. Además de esto, la objeción, según la primera lectura, atribuye al fatalista la creencia de que, a diferencia de los sucesos futuros, los cuales son necesarios por ser inevitables, los sucesos presentes son contingentes, pues pueden no darse. De esto se sigue que los sucesos futuros están subdeterminados por su causa, si por esto se entiende que el suceso ocurrirá aun cuando no ocurra su causa: Abel morirá asesinado aun cuando no sea Caín quien lo asesine. La segunda lectura, en cambio, atribuye al fatalista la creencia de que los sucesos futuros y los presentes son igualmente necesarios. En el ejemplo, el que Caín asesine a Abel es tan necesario como el que Abel muera asesinado. Sin embargo, en un sentido de "subdeterminación" más débil que el anterior, también en este caso los sucesos futuros están subdeterminados por su causa presente. En efecto, por "subdeterminación" también puede entenderse que el efecto sí ocurrirá necesariamente aunque no sea en virtud de su causa (la cual también tiene que darse por necesidad) y esto es justamente lo que se afirma en (2).

Por consiguiente, para evaluar la fuerza de la objeción necesitamos abordar dos problemas diferentes: (a) ¿acepta realmente 
el fatalismo la contingencia de los sucesos presentes, a la vez que niega esta contingencia en el caso de los sucesos futuros, como supone la primera lectura? (b) ¿se sigue realmente del fatalismo que no existe ninguna relación causal explicativa entre sucesos futuros y presentes, como parece asumirse en la segunda lectura? La respuesta a la primera pregunta debe ser "no" si el tipo de fatalismo en cuestión es mínimamente coherente. En efecto, dado que en el pasado el presente estaba en el futuro, el presente estuvo predeterminado del mismo modo en que ahora lo está el futuro. Es por ello que un fatalismo mínimamente coherente tiene que asumir que el presente y el futuro son análogos en cuanto a su comportamiento modal, lo cual explica por qué la respuesta a la primera pregunta debe ser negativa. En todo caso, el fatalismo estoico de Crisipo claramente supone esto. ${ }^{18}$ Por lo tanto, su fatalismo es inmune a la objeción, si la preocupación de Aristóteles, Cicerón y Alejandro al formularla es que no habría una analogía entre el presente y el futuro en cuanto a su comportamiento modal.

Ahora bien, si la preocupación es simplemente que, habiendo una analogía entre presente y futuro, los sucesos futuros no se darían debido a los presentes, la objeción cobra fuerza, pues pone la carga de la prueba del lado del fatalista, quien se ve obligado a mostrar que, a pesar de ser inevitables, los sucesos futuros sí dependen causalmente de los presentes y están completamente determinados por ellos. Crisipo abordó este problema diseñando una teoría de sucesos "codestinados" (confatalia). ${ }^{19}$ No es éste el lugar para emprender una revisión detallada de la teoría, pues otros ya la han emprendido exitosamente. ${ }^{20}$ Pero su idea central es que muchos sucesos futuros

\footnotetext{
${ }^{18}$ Una versión coherente del fatalismo puede aceptar que tanto los sucesos futuros como los pasados son igualmente contingentes en algún sentido. El propio Crisipo acepta esto. Lo que amenaza la coherencia es la falta de analogía entre presente y futuro.

${ }^{19}$ Véase praep. ev. 6.8.25-29 (SVF 2.998; LS 62F). Cfr. F 30 y Cels. 342, $62-65$.

${ }^{20}$ Véanse especialmente Rist 1969, cap. 7, Reesor 1978, Sorabji 1980, cap. 14; LS, cap. 55; Sharples 1991, ad loc.; Sedley 1993. El último y más completo tratamiento de esta teoría es el capítulo 5 de Bobzien 1998.
} 
están "codestinados" con sucesos presentes, donde la noción de suceso codestinado puede definirse como sigue:

(C) un suceso futuro predeterminado $F$ está codestinado con un suceso presente $P$ si, y sólo si, está predeterminado no sólo $F$, sino también el hecho $F P$ que consiste en que $F$ se da porque se da $P$.

De este modo, si $F$ está codestinado con $P$, para que se dé $F$ es condición necesaria que se dé $F P$ y, por lo tanto, el que exista una relación explicativa entre $F$ y $P$. Para retomar el ejemplo que figura en Cicerón, un caso concreto de suceso codestinado sería precisamente el que yo me reponga de la enfermedad. Este suceso estaría codestinado con el que yo llame al médico y siga sus prescripciones. En este caso, el hecho complejo FP sería que yo me repusiera de la enfermedad porque llamo al médico y sigo sus prescripciones. De este modo, y contrariamente a lo que podrían estar suponiendo Aristóteles, Cicerón y Alejandro, no se sigue necesariamente del fatalismo que el futuro sea causalmente independiente del presente. La postura de Crisipo es de hecho la opuesta. En esto radica la diferencia principal entre el fatalismo crisipiano y el fatalismo no causal que podrían estar atacando Aristóteles, Cicerón y Alejandro. Según Crisipo, aquello que suceda o sea el caso en un momento dado depende entera y causalmente de lo que sucede o es el caso en momentos anteriores. Para citar un texto clave de Plutarco sobre Crisipo, "las cosas que se dan primero son causas de las cosas que se dan después de ellas y, en este sentido, todas las cosas están unidas entre ellas" ( $\tau \widetilde{\omega} \nu \pi \rho \omega ́ \tau \omega \nu$

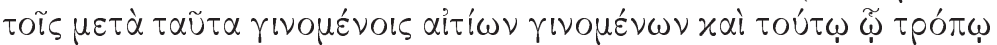

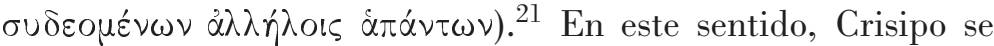
opone no sólo al fatalista incoherente, quien supone que todo estado o suceso está subdeterminado en un sentido fuerte por su causa, sino también al fatalista no causal, quien sostiene que no existe una relación explicativa real entre un estado o suceso

${ }^{21}$ Cfr. St. rep., 1055f-1056a. 
y su causa y, por ello, también supone que todo estado o suceso está subdeterminado por su causa, aunque en un sentido más débil que el anterior.

Ahora bien, ¿por qué debería haber sucesos codestinados en el sentido de Crisipo? ¿Acaso Crisipo no está simplemente suponiendo, en contra del adversario antifatalista, que los hay? La respuesta es negativa. De hecho, Crisipo ofreció una razón sustantiva para su existencia. Detengámonos en un texto del epicúreo tardío Diogeniano, contenido en la Preparación al Evangelio de Eusebio (Praep. ev. 6.8.28), que es clave para entender la teoría de los confatalia.

$<\mathrm{a}\rangle$ Pues, dice [Crisipo], como en el caso siguiente: $\langle\mathrm{b}\rangle$ si alguien afirmara que el boxeador Hegesarco saldrá de la pelea completamente ileso, sería absurdo esperar que peleara sin levantar un brazo porque está predestinado que saldrá ileso de la pelea $(<c>$ pues el que hizo la afirmación dijo esto debido a la excelente guardia de Hegesarco en contra de los golpes); $\langle d\rangle$ así también en todos los demás casos. ${ }^{22}$

Interpreto la afirmación en $<c>$ como sigue: Hegesarco no estaría predeterminado a salir ileso de la pelea de box si peleara sin levantar un brazo, porque no se puede afirmar "el boxeador Hegesarco saldrá de la pelea completamente ileso" sin que esto signifique "saldrá ileso debido a su excelente guardia en contra de los golpes", ${ }^{23}$ lo cual presupone el uso de los brazos. La primera oración expresa el suceso codestinado $F$ (que Hegesarco salga ileso), mientras que la segunda expresa el hecho complejo $F P$, que consiste en que sale ileso $(F)$ debido a su excelente

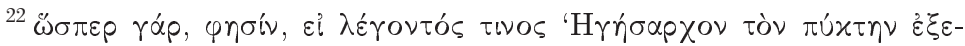

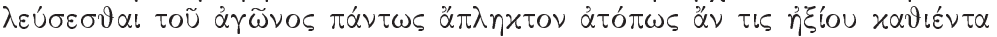

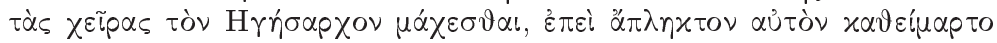

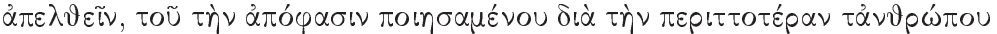

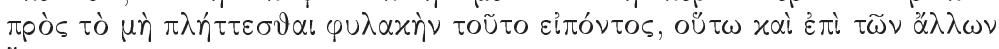
है $\chi \varepsilon เ$.

${ }^{23}$ Aunque el término "signifique" no figure en el texto de Diogeniano, Crisipo se refiere claramente a la implicación — sugerida por la preposición

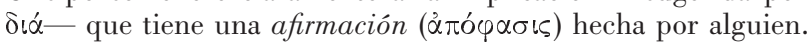


guardia $(P)$. Según Crisipo, la proposición que expresa $F P$ estaría contenida, por decirlo así, en el significado de la proposición que expresa $F$. De este modo, la razón por la cual $F$ no puede estar predeterminado si $P$ no lo está (la razón por la cual $F$ está codestinado con $P$ ) es simplemente conceptual. Algunas proposiciones que expresan sucesos futuros presuponen conceptualmente proposiciones que vinculan explicativamente estos sucesos con otros presentes. Para saber qué sucesos futuros están codestinados con sucesos presentes, se requiere, pero basta, un análisis de los conceptos que se emplean para expresarlos.

Si queremos apreciar la fuerza de la réplica de Crisipo, es necesario tomar en cuenta el contexto dialéctico específico al cual pertenece. A nosotros, sus lectores de principios del siglo XXI, tal vez podrá parecernos que no existe realmente ningún vínculo conceptual o analítico entre, por una parte, salir ileso de una pelea de box y, por otra, emplear una guardia imbatible contra los golpes del adversario. Nos resistiremos a pensarlo si creemos, por ejemplo, que en una pelea de box lo segundo no es la única causa posible de lo primero. Sin embargo, es importante observar que tanto para Crisipo como para su oponente

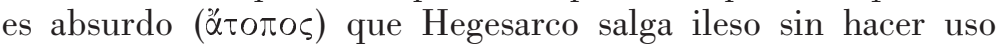
de sus brazos. En efecto, la oración "que [Hegesarco] peleara sin levantar un brazo porque está escrito que saldrá ileso de la pelea" expresa lo que este oponente típicamente identificaría como una consecuencia ineludible pero inaceptable del fatalismo. Ahora bien, ¿por qué les parece absurdo? La respuesta a esta pregunta figura en las reglas mismas que regían las peleas de box del siglo III a.C. Según estas reglas, es imposible que un luchador salga ileso a menos que su guardia sea tan efectiva que le permita cubrirse de todos los golpes de su adversario. ${ }^{24}$ En otras palabras, el concepto de box del siglo III a.C. se define por ciertas reglas y éstas estipulan que un luchador sólo puede evitar los golpes de su adversario (y, con ello, salir ileso de la pelea) manteniendo la guardia. De este modo, el carácter conceptual o analítico de la relación que une salir ileso de una pelea y emplear una guardia imbatible contra los golpes del adversario

${ }^{24}$ Véase Poliakoff 1987, pp. 80-85 y 141-142. 
es relativo al concepto específico de pelea usado por Crisipo y su oponente. La estrategia de Crisipo consiste en explotar este vínculo, reconocido por su oponente, para elaborar su propia noción fatalista de suceso codestinado, argumentando que el suceso futuro está codestinado con el presente porque existe un vínculo explicativo, también reconocido por el oponente, entre ambos. El hecho de que este vínculo explicativo sea relativo a los conceptos específicos que el oponente emplea indica que, según la teoría de los confatalia planteada por Crisipo, el que dos sucesos estén codestinados, o no, depende de qué conceptos se usen para describirlos y, por ende, un suceso está codestinado con otro siempre bajo ciertas descripciones.

Conviene agregar algo que me limité a sugerir líneas atrás. Según Crisipo, hay sucesos futuros, a los que califica de simples (simplex), los cuales están predeterminados sin estar codestinados con ningún suceso presente en especial. Un ejemplo de ello sería que moriré algún día. ${ }^{25}$ En efecto, este suceso no está codestinado con ningún suceso presente en especial, pues si bien lo que hago en el presente puede determinar el modo, e incluso el momento, en que muera, la explicación del hecho de que moriré (a diferencia de cómo lo haré) no se agota en ninguna de las acciones que llevo a cabo en el presente.

Sobre la base del argumento anterior, Crisipo también está en la medida de hacer justicia al supuesto que Aristóteles nos atribuye a todos, a saber, que "si hacemos esto, aquello sucederá, pero si no lo hacemos, entonces no sucederá". Supongamos que nosotros realizamos una acción $\psi$ y creemos que esta acción dará como resultado un suceso $\varphi$ ("si hacemos esto, aquello sucederá": $\psi \rightarrow \varphi$ ). De acuerdo con Aristóteles, esta creencia entraña otra creencia, a saber, que $\varphi$ no sucedería a menos que hagamos $\psi$ ("si no lo hacemos, entonces no sucederá": $\neg \psi \rightarrow$ $\neg \varphi$, que en contraposición es equivalente a $\varphi \rightarrow \psi$. Pero es

${ }^{25}$ El ejemplo de Crisipo según Cicerón ( $c f r . F 30$ ) no es este hecho sino el de que Sócrates morirá cierto día ("morietur illo die Socrates"), lo cual, si nos atenemos a la explicación que hemos dado de la teoría de Crisipo, parecería constituir más bien un suceso codestinado. Estudiosos recientes han intentado explicar en qué sentido este hecho podría ser simplex. Cfr. Sedley 1993, pp. 315-319 y Bobzien 1998, pp. 217-221. 
precisamente esto en lo que Crisipo hace hincapié si $\varphi$ está codestinado con $\psi$, pues si efectivamente lo están, $\varphi$ presupone conceptualmente a $\psi$. Por lo tanto, la teoría de los confatalia de ningún modo quebranta nuestra suposición de que nuestras acciones no son superfluas para producir los resultados que de hecho producen; al contrario, le brinda una fundamentación filosófica. En efecto, una consecuencia de la teoría de los confatalia es que la causa de un estado o suceso codestinado no es sólo una condición suficiente para que este estado o suceso ocurra, sino también una condición necesaria para que lo haga. Esto, a su vez, excluye que un estado o suceso codestinado pudiera estar subdeterminado por su causa.

\section{Fatalismo incoherente, fatalismo no causal y fatalismo causal}

El objetivo de esta última sección es hacer una recapitulación de lo dicho hasta ahora entre los distintos tipos de fatalismo y, sobre esa base, extraer algunas conclusiones filosóficas en torno a la naturaleza del fatalismo de Crisipo.

Son tres las formas de fatalismo de las cuales nos hemos ocupado. Una de ellas es la que quizá tienen en mente Aristóteles, Cicerón y Alejandro de Afrodisia al atacar el fatalismo, y que se podría expresar del modo siguiente:

(I) Para un suceso presente $P$ y un suceso futuro predeterminado $F$ tales que $P$ y $F$ se relacionan aparentemente de modo causal, $F$ habrá de darse aun cuando $P$ no se diera y $P$, a diferencia de $F$, puede no darse.

Esta versión del fatalismo es incoherente pues implica que el futuro y el presente no se comportan modalmente de la misma manera: este último es contingente mientras que el primero es necesario por ser inevitable. Esto representa una incoherencia porque el presente estuvo alguna vez en el futuro $\mathrm{y}$, por lo mismo, es necesario si el futuro en general lo es.

La segunda forma de fatalismo que hemos examinado es de tipo no causal, pues supone que los sucesos futuros no guardan ningún vínculo causal explicativo con los sucesos presentes. 
Conforme a cierta lectura de la objeción de Aristóteles, Cicerón y Alejandro, es esta forma de fatalismo, y no la primera, el blanco de su ataque. El fatalismo no causal se define de la siguiente manera:

(NC) Para un suceso presente $P$ y un suceso futuro predeterminado $F$ tales que $P$ y $F$ se relacionan aparentemente de modo causal, si bien $P$ y $F$ son necesarios, $F$ no se dará porque se da $P$ : no existe realmente una relación causal explicativa entre $F$ y $P$.

La tercera forma de fatalismo es la que defiende Crisipo, quien la distingue explícitamente de (NC). Se trata de una forma causal de fatalismo, pues argumenta a favor de un vínculo causal explicativo entre futuro y presente, sobre la base de consideraciones conceptuales:

(C) Para un suceso presente $P$ y un suceso futuro predeterminado $F$ tales que $P$ y $F$ se relacionan aparentemente de modo causal, tanto $F$ como $P$ son necesarios y el vínculo causal explicativo entre $F$ y $P$, en virtud de que están codestinados, es conceptualmente necesario.

Contra (I), tanto $(\mathrm{NC})$ como $(\mathrm{C})$ son coherentes pues coinciden en que el presente y el futuro se comportan modalmente de la misma forma. Con ello, logran evitar la incoherencia a la cual se ve llevada (I). La diferencia entre $(\mathrm{NC})$ y $(\mathrm{C})$ estriba en que, por contraste con $(\mathrm{C}),(\mathrm{NC})$ sostiene que el futuro no se relaciona de modo causal con el presente.

Podemos entonces ilustrar el lugar que ocupa el fatalismo causal de Crisipo dentro del mapa del fatalismo por medio del esquema de la página 23.

Un tipo de fatalismo no causal es aquel al que podríamos calificar de "lógico". El fatalismo lógico afirma que una proposición que se refiere al futuro es necesaria si es verdadera en el presente, e imposible si es falsa. ${ }^{26}$ Sostiene, asimismo, que el principio de bivalencia se aplica a todas las proposiciones

${ }^{26}$ Esto corresponde a las dos tesis $\left(\mathrm{P}_{n}\right)$ y $\left(\mathrm{P}_{i}\right)$ que mencioné en la sección 1. 
que se refieren el futuro. El fatalismo lógico es fatalista porque argumenta que el futuro está fijado de antemano, y es lógico porque pretende alcanzar esta conclusión únicamente sobre la base del principio lógico de bivalencia y de las tesis lógicas $\left(\mathrm{P}_{n}\right)$ y $\left(\mathrm{P}_{i}\right)$. Conviene detenernos en esta clase de fatalismo porque algunos estudiosos lo han identificado como el blanco de los argumentos antideterministas que Aristóteles plantea en int. $9,{ }^{27}$ y en la antigüedad ha sido confundido con el tipo de fatalismo que Crisipo defiende. ${ }^{28}$

El carácter no causal del fatalismo lógico se hace patente si consideramos un caso concreto. Si es verdadera ahora la proposición $S$ sucede en $t$, donde $t$ es un momento futuro, es necesario ahora que $S$ suceda en $t$ porque, pase lo que pase de ahora hasta entonces, $S$ habrá de suceder en $t$. El fatalista lógico no afirma la posibilidad de que no suceda lo que debe suceder de ahora hasta entonces, pues, según él, todo suceso que se da es necesario. La idea es, más bien, que $S$ habrá de suceder en $t$ aun cuando per impossibile no pasara ni lo que está pasando ni lo que va a pasar de ahora hasta entonces; por consiguiente, continúa el argumento, el hecho de que $S$ suceda en $t$ no se explica ni por lo que está ahora ni por lo que vaya a pasar de aquí hasta entonces. La restricción 'per impossibile' es, por lo tanto, fundamental porque, como ya vimos, el fatalista lógico no sostiene que sea contingente lo que de hecho sucede. La función de la situación contrafáctica es simplemente hacer hincapié en que los sucesos del futuro no dependen causalmente de los sucesos anteriores a ellos.

En consecuencia, y para concluir, es muy marcada la diferencia entre la posición de Crisipo y la del fatalista lógico. Ambos coinciden en que el principio de bivalencia se aplica a las proposiciones que se refieren al futuro, y también en que el futuro ya está fijado por necesidad; pero difieren

${ }^{27}$ Cfr. Anscombe 1956, Williams 1980 y Lowe 1980. A no ser por el pasaje de int. 9 que cité en la sección anterior (18b30-31), el cual puede sugerir que el blanco del ataque de Aristóteles es el fatalismo incoherente (I), el blanco de int. 9 parece ser efectivamente el fatalismo lógico que acabo de describir.

${ }^{28}$ Cfr. Cicerón, F 28. 


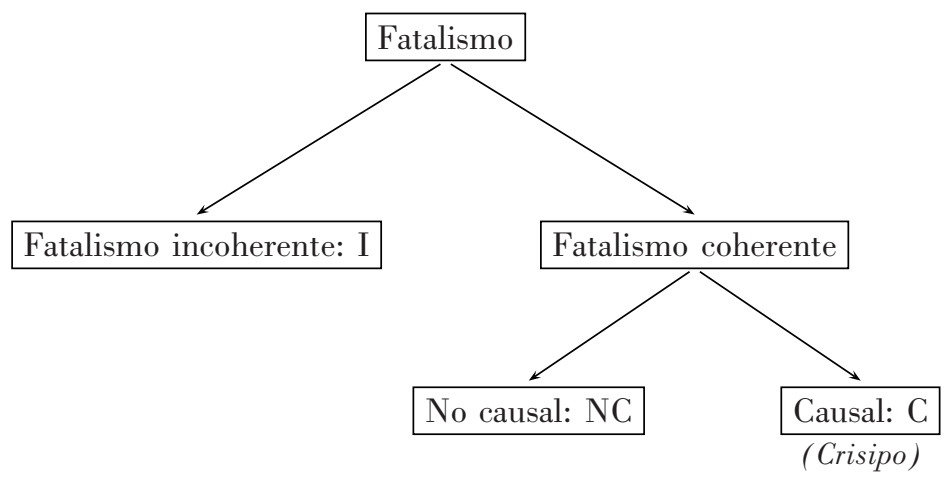

en un aspecto fundamental. En el argumento de la bivalencia de Crisipo recogido por Cicerón, la parte más sustantiva de la inferencia concluye la verdad anticipada de una proposición a partir de la existencia de una causa para el suceso que figura en la proposición. En ningún momento de ese argumento se infiere la necesidad causal del suceso a partir de la verdad de la proposición, como de hecho se inferiría por medio de las tesis $\left(\mathrm{P}_{n}\right)$ y $\left(\mathrm{P}_{i}\right)$, que el fatalista lógico acepta, pero Crisipo rechaza. Como dije antes, no cabe duda de que Crisipo piensa que el futuro ya está fijado, en el sentido de que lo que ha de suceder está predestinado y, por lo tanto, ya es necesario. Pero esta conclusión se alcanza dando otro paso en la argumentación, el cual, como expliqué en la introducción, se apoya en la tesis de que las causas son suficientes para su efecto (no nos ocuparemos de ella en este trabajo). De este modo, Crisipo no pretende que la tesis de la verdad anticipada implique por sí sola la necesidad del suceso futuro correspondiente. El texto del De Fato de Cicerón es engañoso cuando, en el párrafo 28, presenta a los estoicos como si infirieran la predeterminación a partir de la verdad 
anticipada. $^{29}$ Los estoicos sí hacen esta inferencia, pero sólo dada la tesis adicional de que toda causa es suficiente, la cual Crisipo también defiende; sin embargo, él no la emplea en el contexto específico de su argumento de la bivalencia, cuyo propósito es simplemente establecer que todo tiene una causa. $^{30}$

\section{BIBLIOGRAFÍA}

\section{Obras antiguas}

Arnim, H.F.A. von (ed.), Stoicorum Veterum Fragmenta $(=$ SVF), Teubner, Leipzig, 1903-1905, 4 vols.

Ax, W. (ed.), Ciceronis de divinatione, de fato, Timaeus $(=F)$, Teubner, Stuttgart, 1965.

Borret, M. (ed.), Origenis contra Celsum (= Cels.), Cerf, París, 19671976.

Bywater, I. (ed.), Aristotelis Ethica Nicomachea $(=$ EN), Clarendon Press, Oxford, 1894 (1991).

Kalbfleisch, C. (ed.), Simplicii in Aristotelis categorias commentarium, G. Reimer, Berlín, 1907 (Commentaria in Aristotelem Grae$c a, 8)$.

Long, H.S. (ed.), Diogenes Laertius: Vitae Philosophorum (= DL), Clarendon Press, Oxford, 1964.

Minio-Paluello, L. (ed.), Aristotelis Categoriae et liber de interpretatione (= int.), Clarendon Press, Oxford, 1949.

${ }^{29}$ Una inferencia que el propio Cicerón no apoya: "Tampoco, si toda proposición es verdadera o falsa, se sigue por esa razón que haya causas inmutables y eternas que eviten que algo suceda de una manera diferente de como de hecho sucederá" (nec si omne enuntiatum aut verum aut falsum est, sequitur ilico esse causas immutabiles easque aeternas quae prohibeant quicquam secus atque casurum sit). Cfr. F 19.

${ }^{30}$ Este artículo se elaboró en el marco de los proyectos PAPIIT IN 401301, CONACYT 40891-H y FIL-002-03 (Universidad de los Andes, Chile), a los cuales quiero agradecer su apoyo. Se presentaron versiones anteriores de él en el Seminario de Investigadores del Instituto de Investigaciones Filosóficas y en el Primer Taller de Filosofía Antigua, organizado también por el Instituto. Estoy muy agradecido con Gabriela Carone, Guillermo Hurtado, Carlos Pereda y Héctor Zagal por sus observaciones, y con Raymundo Morado y Claudio Veloso por sus valiosísimas réplicas al trabajo. 
Mras, K. (ed.), Eusebii praeparatio evangelica (= praep. ev.), Akademie-Verlag, Berlín, 1954-1956.

Mutschmann, H. y J. Mau (eds.), Sextus Empiricus: Adversus Mathematicos $(=M)$, Teubner, Leipzig, 1914-1961.

Pohlenz, M. y R. Westman (eds.), Plutarchii de Stoicorum repugnantiis (= St. rep.), Teubner, Leipzig, 1952.

Wachsmuth, C. (ed.), Ioannis Stobaei Anthologii libri duo priores qui inscribi solent Eclogae physicae et ethicae $(=E)$, s/e, Berlín, 1884, 5 vols.

\section{Obras modernas}

Anscombe, G.E.M., 1956, "Aristotle and the Sea-Battle", Mind, vol. 65, pp. 1-15. (Reimpreso en J. Smart (comp.), Problems of Space and Time, Macmillan, Nueva York, 1964, pp. 43-57.)

Bobzien, S., 2002, "The Development of Modus Ponens in Antiquity: From Aristotle to the 2nd Century AD", Phronesis, vol. 47, pp. 359-394.

1999, "Chrysippus' Theory of Causes" en K. Ierodiakonou (comp.), Topics in Stoic Philosophy, Clarendon Press, Oxford.

-, 1998, Determinism and Freedom in Stoic Philosophy, Clarendon Press, Oxford.

Bosley, R., 1977, “An Interpretation of On Int 9", Ajatus, vol. 37, pp. 29-40.

Duhot, J.J., 1989, La Conception stoücienne de la causalité, Vrin, París, 1989.

Fine, G., 1984, "Truth and Necessity in De Interpretatione 9", History of Philosophy Quarterly, vol. 1, pp. 23-47.

Frede, M., 1987, "The Original Notion of Cause", Essays in Ancient Philosophy, Clarendon Press, Oxford. (Publicado originalmente en M. Schofield, M. Burnyeat y J. Barnes, Doubt and Dogmatism, Clarendon Press, Oxford, 1980.)

Gaskin, R., 1995, The Sea Battle and the Master Argument. Aristotle and Diodorus Cronus on the Metaphysics of Future, Walter de Gruyter, Berlín/Nueva York.

Hintikka, J., 1973, Time and Necessity, Oxford University Press, Oxford.

- 1964, "The Once and Future Sea-Fight", The Philosophical Review, vol. 73, pp. 461-489.

Long, A.A. y D.N. Sedley, 1987, The Hellenistic Philosophers (= LS), Cambridge University Press, Cambridge. 
Lowe, M., 1980, "Aristotle on the Sea Battle: a Clarification", Analysis, vol. 40, pp. 55-59.

Lukasiewicz, J., 1967, “On Determinism” en S. McCall (comp.), Polish Logic 1920-1939, Clarendon Press, Oxford.

Mackie, J.L., 1965, "Causes and Conditions", American Philosophical Quarterly, vol. 65, no. 2, pp. 245-264. (Reimpreso en E. Sosa (comp.), Causation and Conditionals, Oxford University Press, Londres, 1975, pp. 15-38; las referencias en el texto son a esta versión.)

McCall, S., 1966, "Temporal Flux", American Philosophical Quarterly, vol. 66, no. 3, pp. 270-281.

Poliakoff, M.B., 1987, Combat Sports in the Ancient World. Competition, Violence, and Culture, Yale University Press, New Haven/ Londres.

Quine, W.V.O., 1953, “On a So-Called Paradox”, Mind, vol. 62, pp. 65-67.

Reesor, M.E., 1978, "Necessity and Fate in Stoic Philosophy" en J.M. Rist (comp.), The Stoics, University of California Press, Berkeley/Los Ángeles/Londres.

Rist, J., 1969, Stoic Philosophy, Cambridge University Press, Cambridge, 1969.

Rorty, A.O. (comp.), 1980, Essays on Aristotle's Ethics, University of California Press, Berkeley/Los Ángeles/Londres.

Ross, W.D., 1924, Aristotle's Metaphysics. A Revised Text with Introduction and Commentary, Clarendon Press, Oxford, 2 vols.

Sedley, D., 1993, "Chrysippus on Psychophysical Causation" en J. Brunschwig y M. Nussbaum (comps.), Passions and Perceptions, Cambridge University Press, Cambridge.

Sharples, R.W., 1991, Cicero, On Fate (De Fato) and Boethius, The Consolation of Philosophy iv.5-7, v (Philosophiae Consolationis), Aris and Phillips, Warminster.

Sorabji, R., 1980, Necessity, Cause, and Blame. Perspectives on Aristotle's Theory, Gerald Duckworth, Londres. (Versión en castellano: Necesidad, causa y culpa. Perspectivas sobre la teoría de Aristóteles, trad. Ricardo Salles, Instituto de Investigaciones FilosóficasUNAM, 2003.)

- 1973-1974, "Aristotle on the Role of Intellect in Virtue", Proceedings of the Aristotelian Society, vol. 74, pp. 107-129. (Reimpreso en A.O. Rorty, 1980, pp. 201-219; las referencias en el texto son a esta versión.) 
Wieland, W., 1979, “Aristoteles un die Seeschlacht”, Berichte zur Wissenschaftsgeschichte, vol. 2, pp. 25-33.

Wiggins, D., 1975-1976, "Deliberation and Practical Reason”, Proceedings of the Aristotelian Society, vol. 76, pp. 29-51. (Reimpreso en A.O. Rorty, 1980, pp. 221-265; las referencias en el texto son a esta versión.)

Williams, C., 1980, "What Is, Necessarily Is, When It Is", Analysis, vol. 40, pp. 127-131.

Recibido el 12 de agosto de 2003; revisado el 3 de agosto de 2004; aceptado el 1 de septiembre de 2004. 